\title{
Auditory Brainstem Responses in Newborns With Complete Cleft Palate and Lip at a Japanese Perinatal Center
}

\author{
Masahiko Kato ${ }^{a}$, Mieko Sato ${ }^{a}$, Shunji Suzukia, b
}

\begin{abstract}
Background: Children with cleft palate sometimes suffer from abnormalities in any part of the ear, especially from the middle ear. It is important to identify hearing loss early by monitoring their auditory sensitivity on a regular basis, in order to minimize complications of abnormal or fluctuating hearing on speech development in patients with cleft palate.
\end{abstract}

Methods: We examined auditory brainstem responses (ABRs) in 13 Japanese newborns with complete cleft palate and lip at $4.1 \pm 2.2$ days after delivery compared with sex-, age- and weight-matched 13 Japanese newborns without cleft palate or lip as control.

Results: Wave I component latency (CL) was longer in ABRs of infants with complete cleft palate and lip than control newborns, while waves III and V CLs and the wave I-V interpeak latency (IPL) were not different.

Conclusions: The current results indicate the presence of congenital conductive hearing disturbance in newborns with complete cleft palate and lip.

Keywords: Complete cleft palate and lip; Auditory brainstem response; Newborn; Japan

\section{Introduction}

Undetected congenital permanent hearing loss impairs de-

Manuscript accepted for publication September 15, 2014

${ }^{a}$ Department of Obstetrics and Gynecology, Japanese Red Cross Katsushika Maternity Hospital, Tokyo, Japan

${ }^{b}$ Corresponding Author: Shunji Suzuki, Department of Obstetrics and Gynecology, Japanese Red Cross Katsushika Maternity Hospital,

5-11-12 Tateishi, Katsushika-ku, Tokyo 124-0012, Japan.

Email:czg83542@mopera.ne.jp

doi: http://dx.doi.org/10.14740/ijcp168w velopment in infants by diminishing their ability to acquire language and cognitive skills at an appropriate age [1]. Children with cleft palate sometimes suffer from abnormalities in any part of the ear, especially from the middle ear. The high incidence of conductive hearing loss from serous effusion associated with serous otitis media in patients with cleft palate is well known [2]. For this reason, it is important to identify hearing loss early by monitoring their auditory sensitivity on a regular basis, in order to minimize complications of abnormal or fluctuating hearing on speech development in patients with cleft palate.

In the current study, therefore, we examined auditory brainstem responses (ABRs) in Japanese newborns with complete cleft palate and lip compared with sex-, age- and weight-matched Japanese newborns without cleft palate or lip at Japanese Red Cross Katsushika Maternity Hospital, Tokyo, Japan.

\section{Materials and Methods}

The protocol for this study was approved by the Ethics Committee of the Japanese Red Cross Katsushika Maternity Hospital. Our hospital is one of the major perinatal centers for high risk pregnancies in Tokyo, Japan.

There were 20 Japanese newborns with cleft palate and/ or lip who were born at Japanese Red Cross Katsushika Maternity Hospital from 2011 to 2013 . Of these, 13 (65\%, three female) newborns born at $38.4 \pm 1.6$ weeks' gestation were complicated by isolated bilateral or unilateral complete cleft palate and lip.

After obtaining the family's consents, in the current study infants were screened with a Neuropack $\mu^{\circledR}$, an ABR sensor at $4.1 \pm 2.2$ days after delivery. All examinations were performed by one experienced examiner (M.S.). Infants who did not pass the ABR were referred to the specialists of hearing loss as previously reported [3]. We also examined the sex-, age- and weight-matched 13 Japanese newborns without cleft palate or lip as control.

Data are presented as mean $\pm \mathrm{SD}$. The Student's $t$-test for continuous variables was used. Differences with $\mathrm{P}<0.05$ were considered significant. 
Table 1. Mean CLs and IPLs in Neonates With and Without Complete Cleft Palate and Lip
Cleft palate and lip $(n=13$, three female $)$
Control $(n=13$, three female)
P-value

\begin{tabular}{lccc}
\hline Wave I CL & $1.72 \pm 0.22$ & $1.48 \pm 0.19$ & 0.007 \\
Wave III CL & $4.68 \pm 0.44$ & $4.39 \pm 0.25$ & 0.051 \\
Wave V CL & $6.85 \pm 0.46$ & $6.60 \pm 0.28$ & 0.107 \\
Wave I-V IPL & $5.11 \pm 0.38$ & $5.13 \pm 0.22$ & 0.871 \\
\hline
\end{tabular}

Data are reported as mean $\pm \mathrm{SD}$. CL: component latency; IPL: interpeak latency.

\section{Results}

There were no significant differences in the birth weights or postconceptional age (PCA) between the two groups (control: $\mathrm{n}=13$ (three female), 2,599 $\pm 515 \mathrm{~g}$ and $39.0 \pm 1.4$ weeks; complete cleft palate and lip: $n=13$ (three female), $2,609 \pm 496 \mathrm{~g}$ and $39.0 \pm 1.6$ weeks).

During the study period, there were no newborns with isolated complete cleft palate and lip who did not pass the ABR. Table 1 shows the mean component latencies (CLs) and interpeak latencies (IPLs) in the two groups of neonates with and without complete cleft palate and/or lip. As shown in Table 1, wave I CL was longer in ABRs of infants with complete cleft palate and lip than control newborns, while waves III and V CLs and the wave I-V IPL were not different.

\section{Discussion}

ABR is an effective noninvasive measure of sensorineural and conductive hearing loss. In ABR, wave I and wave II are generated from the eighth nerve and cochlea on the ipsilateral side, while waves III and V are generated from a complex interaction of both contralateral and ipsilateral brainstem anatomy. Thus, conductive hearing loss is typically indicated be a delayed wave I CL or a poorly defined wave I [4].

Hearing loss is a well-known complication of cleft palate, but the magnitude of this problem is not generally appreciated [5]. A lot of attention has been paid to the development of a competent velopharyngeal port and normal facial development in these children. This concern with the production of normal speech and the prevention of facial deformity has diverted attention from a common but unfortunately ignored complication of hearing loss [5].

The abnormal reflux of food and fluid into the nasal cavity can set up chronic inflammatory changes such as edema, hypertrophy, obstruction and secondary middle ear disease [5]. These inflammatory changes result in otitis media lead- ing to conductive hearing loss in patients with cleft palate [5]. However, the current results indicate the presence of congenital conductive hearing disturbance in newborns with complete cleft palate and lip before the development of serous otitis media. The current results seemed to be contrary to the previous study by Yang et al [6] that there was no significant difference in ABR (all measurements including wave I).

Based on some previous studies [2, 7], some possible mechanisms leading to the current results can be proposed. In an earlier review by Young and Deskin [2], the eustachian tube dysfunction in patients with cleft palate was observed to be due to an abnormal insertion of the levator and tensor veli palatini muscles into the posterior margin of the hard palate. Although the incidence of eustachian tube dysfunction has been reported to decrease with increasing age and in many cases normal eustachian tube function has been observed to develop by mid adolescence [2], routine serious follow-up examinations of the ears are needed in patients with complete cleft palate from neonatal period until the age of mid adolescence.

In conclusions, the current results indicate the presence of congenital conductive hearing disturbance in newborns with complete cleft palate and lip.

\section{References}

1. Erenberg A, Lemons J, Sia C, Trunkel D, Ziring P. Newborn and infant hearing loss: detection and intervention.American Academy of Pediatrics. Task Force on Newborn and Infant Hearing, 1998- 1999. Pediatrics. 1999;103(2):527-530.

2. Young G, Deskin R. Cleft Lip and Palate. 1998; http:// www2.utmb.edu/otoref/Grnds/Cleft-lip-palate-9801/ Cleft-lip-palate-9801.htm.

3. Sato M, Suzuki S. Referral rates and costs of our modified two-step newborn hearing screening program at a 
Japanese perinatal center. Int J Clin Pediatri. 2014;3:3539.

4. Squires N, Ollo C, Jordan R. Auditory brain stem responses in the mentally retarded: audiometric correlates. Ear Hear. 1986;7(2):83-92.

5. Sharma RK, Nanda V. Problems of middle ear and hearing in cleft children. Indian $\mathrm{J}$ Plast Surg. 2009;42(Suppl):S144-148.
6. Yang FF, McPherson B, Shu H, Xiao Y. Central auditory nervous system dysfunction in infants with nonsyndromic cleft lip and/or palate. Int J Pediatr Otorhinolaryngol. 2012;76(1):82-89.

7. Kawase K, Yamazaki T, Masuda K, Uenaka Y, Yasaki T. Auditory brainstem responses in newborns with cleft palate and/or lip (in Japanese). Jap J Pediatr 1994;47:17491753. 